\title{
Possible hidden hazards of mass vaccination against new influenza A/H1N1: have the cardiovascular risks been adequately weighed?
}

\author{
Sucharit Bhakdi · Karl Lackner • Hans-Wilhelm Doerr
}

Published online: 23 October 2009

(C) Springer-Verlag 2009

\begin{abstract}
Programs for vaccination against the new influenza $\mathrm{A} / \mathrm{H} 1 \mathrm{~N} 1$ targeting many hundred million citizens in Europe and the USA are to be launched in the fall of this year. The USA is planning to employ a non-adjuvanted vaccine, whereas European nations are opting for inclusion of MF59, the adjuvant contained in an alternative seasonal flu vaccine, or the related adjuvant AS03 that is contained in a recently developed $\mathrm{H} 5 \mathrm{~N} 1$ vaccine. We draw attention to unappreciated hazards of using adjuvanted vaccine in Europe. Evidence from animal experiments in conjunction with clinical epidemiological data indicates that, quite irrespective of cause, stimulation of the immune system may accelerate atherogenesis. Application of adjuvanted flu vaccines to individuals at risk may therefore aggravate the course of underlying atherosclerotic vessel disease with all the clinical consequences. The same may hold true for other widespread diseases that are propelled by deregulated immune mechanisms. Safety trials conducted to date have not specifically taken these possible side effects into account, and unexpected serious adverse effects thus may follow in the wake of a general vaccination program.
\end{abstract}

\section{S. Bhakdi $(\square)$}

Institute of Medical Microbiology and Hygiene,

University Medical Center, Augustusplatz,

55101 Mainz, Germany

e-mail: sbhakdi@uni-mainz.de

\section{K. Lackner}

Department of Clinical Chemistry and Laboratory Medicine,

University Medical Center, Langenbeckstrasse 1,

55131 Mainz, Germany

\section{H.-W. Doerr}

Department of Medical Virology,

Johann Wolfgang Goethe-University Clinic,

Paul-Ehrlich-Str. 40, 60596 Frankfurt/Main, Germany
A prudent consequence would be to establish careful survey systems alongside with mass application of new adjuvanted vaccines, or to hold mass vaccination in reserve for use only in situations of true need, such as would arise with the emergence of a more virulent new H1N1 virus strain, or to use non-adjuvanted vaccines in individuals who are potentially at risk for adverse side effects.

\section{Background}

The current flu pandemic that originated in Mexico in April 2009 has triggered a global reaction of unprecedented dimension in science and society $[1,2]$. Fortunately, the H1N1 virus has not yet transformed itself into the dreaded killer, although well over 300.000 infections have been documented [3]. The probability that such a transformation will ever take place is thus obviously small, but a single event could have dire consequences. With high priority, vaccination programs were accordingly conceived and are already on the verge of implementation [4, 5]. Vaccination is also expected to reduce the death toll and costs for hospitalization incurred by H1N1. Somewhat oddly, the same arguments have not been made to implement a similar vaccination program against seasonal flu, which would appear reasonable. Instead, at least 1.000.000.000 Euros are planned to be spent to vaccinate one-third of the German population against $\mathrm{H} 1 \mathrm{~N} 1$, and troubled discussions are ongoing behind the scenes as to how this is to be financed.

\section{The vaccines}

Non-adjuvanted vaccines are alike to conventional seasonal flu vaccines. Antigen content can be reduced through 
addition of adjuvant, and a number of adjuvanted flu vaccines have accordingly been developed [6]. One is modeled after Fluad $^{\circledR}$, the vaccine developed over 10 years ago that contains the adjuvant MF59 and that is also being employed worldwide mainly in subjects over 65 years of age. About 45 million doses of Fluad ${ }^{\circledR}$ have been used over the last 10 years with no serious side effects being recognized to date. A number of arguments speak for inclusion of MF59 or a related agent in H1N1 vaccines, and many European Nations including Germany have opted for this choice. Until recently, the US has favored employment of non-adjuvanted vaccine, but discussions on this issue are currently ongoing, and a final decision is still pending. The question whether adjuvanted vaccine needs to be applied once or twice is also not definitely settled. A first report indicates that a single application may suffice in adults [7].

MF59, a squalene oil-in-water emulsion with yet poorly delineated mechanism of action, appears to target monocytes, macrophages and dendritic cells via mechanisms distinct from those mediated from danger signals. MF59 induces macrophage recruitment to the injection site and appears to promote uptake of antigen by macrophages and dendritic cells. In vitro experiments indicate that MF59 induces a range of chemoattractants but not classical proinflammatory cytokines in human monocytes and monocyte-derived macrophages [8]. However, injection of adjuvanted flu vaccine frequently causes local pain and occasionally fever [7], an indication that proinflammatory cytokines are generated in appreciable amounts. MF59adjuvanted flu-vaccines have been applied in young children but there is little experience with their use in pregnant women. Another adjuvanted H1N1 vaccine is based on an H5N1-vaccine developed by GSK [9]. The AS03 adjuvant is very similar to MF59. In addition to squalene, the GSK vaccine also contains the non-ionic detergent polysorbate 80 (Tween 80), with uncharacterized pharmacokinetic and immunological properties, and alpha-Tocopherol. Studies with vaccines containing AS03 in infants, young children or pregnant women have not been published. Current clinical studies are being conducted with children aged 3-17.

\section{Hidden dangers of adjuvanted H1N1 vaccines}

While the possible risks of the current vaccination program have been the subject of countless discussions, one potential hazard may still not have been adequately addressed, and to this attention is drawn here. It is today widely held that immunopathological mechanisms underlie chronic diseases of natures so diverse that no common denominator could have come to mind prior to the advent of modern immunology. A major conceptual hurdle that needed to be taken is related to the lack of recognizable specificity of such self-destructive processes. Now, it is realized that innate, unspecific immune mechanisms rival adaptive immunity in fueling the pathology of some of the most widespread diseases of mankind including atherosclerosis, inflammatory bowel disease, demyelinating diseases and non-infectious arthritis. These examples are given to highlight their diversity; the list is ever growing. ${ }^{1}$

Paired with this is the emerging concept that such pathological processes sometimes have their roots in physiological events that normally serve meaningful biological purposes. Atherosclerosis is a case in point. Mobilization of innate immune components to intimal lipids possibly represents a physiological means to clear tissues of cholesterol, whose poor solubility necessitates its removal by macrophages. Clinical manifestations of atherosclerotic vessel disease ensue only when pathological late lesions evolve as the cholesterol removal machinery breaks down through overload. Lesional macrophages then cease to execute their physiological function in a quiescent manner and detrimental effects follow with all the known clinical consequences.

An aspect of prime interest relates to the question of whether such processes can be influenced by accompanying, primarily unrelated immunological events. Again, atherosclerosis is a disease for which an emphatic affirmative answer must be given. It is today disputed that flawed regulation of innate and adaptive immunity can accelerate and aggravate atherogenesis. While debates abound on the relative importance of innate versus adaptive mechanisms, ${ }^{2}$ they do not detract from this basic tenet, which satisfactorily accounts for the long-known clinical observation that lesion pathology is fuelled by such diverse conditions as acute and chronic infections or stress, smoking and diabetes.

That immune activation occurring at sites remote from the lesions themselves should be able to influence their progression likely derives from the mobility and lack of specificity of monocyte/macrophages. Thus, while these cells usually perform their physiological, cholesterolscavenging function in the absence of inflammation, homeostasis is labile and can be disrupted when concomitant external or internal danger signals arrive on the scene. This concept of immune-mediated collateral damage has been borne out by straightforward experiments. For example, rabbits on hypercholesterolemic diet given low dose injections of endotoxin that caused brief rises of only $1{ }^{\circ} \mathrm{C}$ in body temperature developed markedly larger

\footnotetext{
1 As of 22.9.2009, PubMed hits for innate immunity and inflammatory bowel disease: 472 ; atherosclerosis: 445 ; demyelinating disease: 402; reactive arthritis: 82 .

${ }^{2}$ PubMed hits for adaptive immunity and atherosclerosis: 106.
} 
atherosclerotic lesions than controls [10]. This is particularly relevant in the present context because it highlights the fact that macrophage stimulation accelerates atherogenesis in the absence of any infection [10, 11]. Toll-likereceptor polymorphisms influence the response of cells to activating stimuli, and weak responses render individuals less prone to atherosclerotic vascular disease [12]. Cigarette smoke provokes a spectrum of proinflammatory effects, which provide a simple explanation for its atherogenic effects. ${ }^{3}$ Advanced glycation end products (AGEs) were originally linked to atherosclerotic cardiovascular disease. Binding of AGEs to their receptor (RAGE) induces sustained inflammatory signal amplification $[13,14]$ and this may be one reason why diabetes accelerates atherogenesis in humans, and why elimination of the receptor retards atherosclerosis in animals [15]. These few examples should suffice to transmit that if, at any stage, vaccination drives macrophages into their inflammatory state, the effects on atherosclerosis will hardly be unpredictable and acute clinical events could be precipitated. Causes might be the adjuvant or another ingredient, a combination of both, or any other inflammatory events provoked by intramuscular injection of the vaccine.

Against this background, the discomforting question arises whether any clinical data exist that would relieve us of these concerns. The simple answer is no. Although millions of individuals have been vaccinated with the adjuvanted flu vaccine, data relating to any of the previous questions have not been systematically acquired. Trials conducted with H1N1 vaccines also do not and cannot address these questions. Such trials would have to be conducted in individuals with identified risk factors, and observations would have to be made over extended periods of time. The latter also holds for healthy young individuals who are to repeatedly receive adjuvanted vaccines over years or decades. That unknown dangers may also be lurking here should be self-evident. To top matters, the GSK flu vaccine with its novel combination of adjuvant and additives has not ever been applied to a large number of recipients. The adjuvant included in the GSK vaccine is AS03 in the same or similar formulation previously employed in the adjuvanted H5N1 influenza vaccine [9]. The safety of that vaccine was assessed in 400 volunteers. Fever developed in 4 of 200 participants who received nonadjuvanted vaccine and in 15 participants who received the adjuvanted vaccine [9]. Adjuvantation markedly increased the incidence of all other registered local and general symptoms, e.g. local redness: $20.5 \%(+)$ vs. $12 \%(-)$; swelling: $18.5 \%(+)$ vs. $6 \%(-)$; muscle aches: $43.5 \%(+)$ vs. $15.5 \%(-)$. If anything, these data should serve to

\footnotetext{
329 PubMed hits for proinflammatory effects of cigarette smoke.
}

nurture rather than to dispel the present concerns. As commented in an overview released by the WHO, evaluation of potential immune-mediated effects of AS03 adjuvanted vaccines is currently limited by the small number of events and unbalanced randomization of subjects [6]. Clearly, the case is open.

A most recent publication might convey the impression that the situation may be more favorable for the MF59 adjuvanted vaccine [16]. A retrospective analysis was compiled from 63 clinical trials conducted between 19921993 and 2007-2008 influenza seasons in Europe, USA, Australia and South America, and data were acquired for a total of 4,115 elderly subjects vaccinated with and 1,753 subjects vaccinated without MF59. There was a tendency toward lower risks of adverse cardiovascular events in the (+) MF59 group. However, demographic data on race were not available for $70-75 \%$ of the enrolled population, and cases of myocardial infarctions were neither given nor compared with the expected numbers. Thus, this study also provides no real answers to the present questions.

Now, it could be argued that the discussed hazards of vaccination are just hypothetical and, given the impossibility of conducting the necessary safety trials, cannot be taken as arguments to impede implementation of the vaccination programs. Vice versa, however, fear that emergence of a hypervirulent H1N1 strain will emerge or that the clinical course of infections with the present virus will dramatically worsen during the winter also rests on conjecture. The argument that mass vaccination could significantly reduce the chances that a hypervirulent strain emerges is difficult to follow, because only a small part of the world population is being targeted for protection.

The possible connection between vaccination of individuals at risk and triggering of life-threatening cardiac events might have just been on the verge of discovery in 2003. During January 24 to May 2, 2003, smallpox vaccine was administered to 36.000 civilians in the USA. Five myocardial infarctions (MI) occurred within 3 weeks, the time frame empirically chosen to define possible side effects, following vaccination in individuals aged 46-65. Five MI were higher than the two that would have been expected in this age group, and approached but did not exceed the upper 95\% predictive interval of 5.4 [17].

If our arguments are followed, the thought that a live vaccine cannot be compared with the present flu vaccine will hardly come to bear. Collateral damage occurring in atherosclerotic lesions will likely be incurred through any vigorous activation of the immune system. This point needs to be reiterated, for only thus can the generality of the present arguments be appreciated. That acute stress situations can precipitate cardiac events is common knowledge, and triggering of the proinflammatory arm of the immune system can be alikened to such a situation. Clinical 
manifestations will, of course, only occur in the few individuals who are on the brink of a cardiovascular event. But consider that the intended number of H1N1 vaccinations in Europe is 10.000 -fold higher than the number of civilian smallpox vaccinees in 2003. Furthermore, individuals with chronic underlying diseases including cardiac and pulmonary patients are among the first to be targeted in the impending anti-H1N1 campaign. It is noteworthy that cardiovascular diseases actually became recognized as contraindications for smallpox immunization during the short course of the 2003 campaign. What a disaster if the forthcoming program should force us in hindsight to realize that the same holds true for flu vaccinations!

It may be argued that a CDC article published in October 2003 explicitly negated a connection between smallpox vaccination and adverse cardiac events [18]. In that retrospective study, the New York City Department of Health and Mental Hygiene analyzed data from NYC death certificates of 1947 , when 6.000 .000 NYC residents $(80 \%$ of the population) were vaccinated against smallpox during the 4 week period after a smallpox outbreak. The conclusion was that there was no increase in cardiac deaths, so those observed after the 2003 campaign were probably unrelated to the vaccine. But what if that study had missed its mark simply because of the lower prevalence of atherosclerosis in the 1947 US population? Furthermore, hypertension was a major underlying cause for cardiac deaths at that time, and this ailment would not have been influenced by vaccination. Thus, the conclusion drawn in the study may actually be clouding perception and concealing the truth from unseeing eyes.

\section{Mass H1N1 vaccination: to do or not to do}

The authors do not question the necessity of developing effective vaccine strategies against H1N1. There is no intent to criticize, and less still to blame any of the decision-makers for the course the program has taken thus far. Quite to the contrary, it is recognized that the preparations undertaken to face the $\mathrm{H} 1 \mathrm{~N} 1$ as well as the $\mathrm{H} 5 \mathrm{~N} 1$ threat set impressive standards for the future [19-21]. Of ultimate importance now is wise timing of the next move.

Foremost, the question to be answered is whether the hazards of conducting mass vaccinations at the present stage might outweigh the expected benefits. One argument in favor of mass vaccination derives from observational studies reporting reductions in all-cause mortality in older persons who had been vaccinated against seasonal flu vs. non-vaccinated individuals [22-28]. At present, however, H1N1-related mortality is very low in Europe, and whether it will rise to the extent observed with seasonal flu in winter simply cannot be predicted.
To quote EMEA (European Medicines Agency): "Public confidence in vaccination programs may only be maintained if it is considered that competent authorities will access the safety of vaccines in a timely and adequate manner and take appropriate action. This includes investigation of rare and unexpected adverse events, increases in the occurrence of known adverse reactions and careful analysis of theoretical concerns". Now, should any of the thoughts expressed here contain just a grain of truth, this cannot but be uncovered by the sheer numeric power of the program. If, in retrospect, vaccination should turn out to have been unnecessary because a virulent H1N1-virus never emerged, consequences for the responsible parties can be foreseen, and public faith in preventive medicine will be shaken to the roots.

Must these risks be taken? The production of sufficient H1N1 vaccines is guaranteed, and the world is now armed to conquer the hypervirulent virus, should it ever emerge. Surveillance programs have been installed to ensure that the new foe is quickly tracked down, and action could then be immediately taken.

So why play out a trump prematurely? Man has already won the first round in the race against this adversary. Why not hold mass vaccination in reserve? Or, if the vaccination program is implemented, would it not be prudent to use non-adjuvanted vaccine in individuals at risk? We have focused on cardiovascular disorders but the arguments would extend to other diseases that harbor immunopathological components. Finally, if the decision is still made to uniformly employ adjuvanted vaccines, careful survey systems should be implemented to clearly establish that the concerns expressed here, in particular the potential of adjuvanted vaccines to precipitate cardiovascular events, are nothing more than theory.

Acknowledgments We thank Dr. Markus Knuf and Dr. Karina Reiß for many helpful suggestions and constructive criticisms. Dr. Markus Knuf forfeited his coauthorship due to a conflict of interest. We also thank Monika Wiedmann for subperb secretarial assistance.

\section{References}

1. Chan M (2009) World now at the start of 2009 influenza pandemic: http:/www.who.int/mediacentre/news/statements/2009/ h1n1_pandemic_phase6_20090611/en/index.html. Statement to the press by WHO Director General Dr Margaret Chan; WHO, 11. Juni 2009

2. WHO (2009) WHO pandemic phase description and main actions by phase. http://www.who.int/csr/disease/influenza/ GIPA3AideMemoire.pdf

3. CDC CfDCaP (2009) 2009 H1N1 Flu: international situation update. http://www.cdc.gov/h1n1flu/updates/international/

4. ACIP (2009) Use of influenza A (H1N1) 2009 monovalent vaccine: recommendations of the Advisory Committee on Immunization Practices (ACIP), 2009. MMWR Recomm Rep 58:1-8 http://www.ncbi.nlm.nih.gov/entrez/query.fcgi?cmd=Retrieve\& $\mathrm{db}=$ PubMed\&dopt $=$ Citation\&list_uids $=19713882$ 
5. Neuzil KM (2009) Pandemic influenza vaccine policy—considering the early evidence. $\mathrm{N}$ Engl $\mathrm{J}$ Med http://www.ncbi.nlm. nih.gov/entrez/query.fcgi? $\mathrm{cmd}=$ Retrieve $\& \mathrm{db}=$ PubMed\&dopt $=$ Citation\&list_uids $=19745213$

6. Reed S (2009) Brief overview of use of oil-in-water emulsions as adjuvants for influenza vaccines. http:/www.who.int/vaccine_ research/documents/Brief_overview_of_use_of_oil-in-water_ emulsions.pdf

7. Clark TW, Pareek M, Hoschler K, Dillon H, Nicholson KG, Groth N, Stephenson I (2009) Trial of influenza A (H1N1) 2009 monovalent MF59-adjuvanted vaccine-preliminary report. N Engl J Med http://www.ncbi.nlm.nih.gov/entrez/query.fcgi?cmd= Retrieve $\& d b=$ PubMed\&dopt=Citation\&list_uids $=19745215$

8. Seubert A, Monaci E, Pizza M, O’Hagan DT, Wack A (2008) The adjuvants aluminum hydroxide and MF59 induce monocyte and granulocyte chemoattractants and enhance monocyte differentiation toward dendritic cells. J Immunol 180:5402-5412

9. Leroux-Roels I, Borkowski A, Vanwolleghem T, Drame M, Clement F, Hons E, Devaster JM, Leroux-Roels G (2007) Antigen sparing and cross-reactive immunity with an adjuvanted rH5N1 prototype pandemic influenza vaccine: a randomised controlled trial. Lancet 370:580-589

10. Lehr HA, Sagban TA, Ihling C, Zahringer U, Hungerer KD, Blumrich M, Reifenberg K, Bhakdi S (2001) Immunopathogenesis of atherosclerosis: endotoxin accelerates atherosclerosis in rabbits on hypercholesterolemic diet. Circulation 104:914-920 http://www.ncbi.nlm.nih.gov/entrez/query.fcgi?cmd=\&db=PubMed \&dopt=Citation\&list_uids $=11514379$

11. Bhakdi S, Lackner KJ, Han SR, Torzewski M, Husmann M (2004) Beyond cholesterol: the enigma of atherosclerosis revisited. Thromb Haemost 91:639-645

12. Kiechl S, Lorenz E, Reindl M, Wiedermann CJ, Oberhollenzer F, Bonora E, Willeit J, Schwartz DA (2002) Toll-like receptor 4 polymorphisms and atherogenesis. N Engl J Med 347:185-192

13. Herold K, Moser B, Chen Y, Zeng S, Yan SF, Ramasamy R, Emond J, Clynes R, Schmidt AM (2007) Receptor for advanced glycation end products (RAGE) in a dash to the rescue: inflammatory signals gone awry in the primal response to stress. J Leukoc Biol 82:204-212

14. Bopp C, Bierhaus A, Hofer S, Bouchon A, Nawroth PP, Martin E, Weigand MA (2008) Bench-to-bedside review: the inflammationperpetuating pattern-recognition receptor RAGE as a therapeutic target in sepsis. Crit Care 12:201

15. Soro-Paavonen A, Watson AM, Li J, Paavonen K, Koitka A, Calkin AC, Barit D, Coughlan MT, Drew BG, Lancaster GI, Thomas M, Forbes JM, Nawroth PP, Bierhaus A, Cooper ME, Jandeleit-Dahm KA (2008) Receptor for advanced glycation end products (RAGE) deficiency attenuates the development of atherosclerosis in diabetes. Diabetes 57:2461-2469
16. Pellegrini M, Nicolay U, Lindert K, Groth N, Della Cioppa G (2009) MF59-adjuvanted versus non-adjuvanted influenza vaccines: Integrated analysis from a large safety database. Vaccine [Epup ahead of print] http://www.ncbi.nlm.nih.gov/entrez/query. fcgi?cmd=Retrieve $\& \mathrm{db}=$ PubMed $\&$ dopt $=$ Citation $\&$ list_uids $=$ 19751689

17. (2003) Update: cardiac-related events during the civilian smallpox vaccination program-United States, 2003. MMWR Morb Mortal Wkly Rep 52:492-496 http://www.ncbi.nlm.nih.gov/ entrez/query.fcgi?cmd=Retrieve $\& \mathrm{db}=$ PubMed\&dopt=Citation \& list_uids $=12809110$

18. (2003) Cardiac deaths after a mass smallpox vaccination campaign-New York City, 1947. MMWR Morb Mortal Wkly Rep 52:933-936

19. Cinatl J Jr, Michaelis M, Doerr HW (2007) The threat of avian influenza A (H5N1). Part I: epidemiologic concerns and virulence determinants. Med Microbiol Immunol 196:181-190

20. Cinatl J Jr, Michaelis M, Doerr HW (2007) The threat of avian influenza A (H5N1). Part IV: development of vaccines. Med Microbiol Immunol 196:213-225

21. Michaelis M, Doerr HW, Cinatl J Jr (2009) Novel swine-origin influenza A virus in humans: another pandemic knocking at the door. Med Microbiol Immunol 198:175-183

22. Ortqvist A, Granath F, Askling J, Hedlund J (2007) Influenza vaccination and mortality: prospective cohort study of the elderly in a large geographical area. Eur Respir J 30:414-422

23. Gross PA, Hermogenes AW, Sacks HS, Lau J, Levandowski RA (1995) The efficacy of influenza vaccine in elderly persons. A meta-analysis and review of the literature. Ann Intern Med $123: 518-527$

24. Jefferson T, Rivetti D, Rivetti A, Rudin M, Di Pietrantonj C, Demicheli V (2005) Efficacy and effectiveness of influenza vaccines in elderly people: a systematic review. Lancet 366:1165-1174

25. Vu T, Farish S, Jenkins M, Kelly H (2002) A meta-analysis of effectiveness of influenza vaccine in persons aged 65 years and over living in the community. Vaccine 20:1831-1836

26. Jansen AG, Sanders EA, Nichol KL, van Loon AM, Hoes AW, Hak E (2008) Decline in influenza-associated mortality among Dutch elderly following the introduction of a nationwide vaccination program. Vaccine 26:5567-5574

27. Puig-Barbera J, Diez-Domingo J, Varea AB, Chavarri GS, Rodrigo JA, Hoyos SP, Vidal DG (2007) Effectiveness of MF59adjuvanted subunit influenza vaccine in preventing hospitalisations for cardiovascular disease, cerebrovascular disease and pneumonia in the elderly. Vaccine 25:7313-7321

28. Warren-Gash C, Smeeth L, Hayward AC (2009) Influenza as a trigger for acute myocardial infarction or death from cardiovascular disease: a systematic review. Lancet Infect Dis 9:601-610 